\title{
ON LENGTH-MINIMIZING STEINER NETWORKS
}

\author{
By Dao Trong Thi and Nguyen HuU Quang
}

\section{Introduction}

Let $\mathrm{M}$ be a set of points in $R^{n}$. The Steiner problem can be stated as follows: to find a network of least length in the class of networks with fixed ends $M$. There are two approaches to this problem. In the first case, the search for an absolutely minimal network is carried out in the class of networks, whose vertices all belong to $\mathbf{M}$. This approach is developed in the papers by Du, Hwang and others (see, for example, [5], [6]). In the second case, the set of vertices of the networks may be larger than the set $M$. The vertices do not belong to $\mathbf{M}$ are called Steiner points and the points in $\mathbf{M}$ are called boundary points. In this paper we study globally minimal network in the second case. By using calibration systems we prove that each locally minimal network is also globally minimal in the class of networks with the same topological type. The method of calibrations was developed in works of Federer, Dao Trong Thi, Lawson, Harvey, Morgan and others (see, for example, [1]-[3]). Calibration systems were used first in [4].

\section{Steiner networks}

Definition 1.1. A Steiner network in $R^{n}$ is any connected complex of one-dimensional simplexes, whose vertices have degree at most three. A Steiner network without vertices of degree two is said to be nondegenerate Steiner network.

Henceforth we shall study only acyclic nondegennerate Steiner networks with boundary points coinsiding with the vertices of degree one. Such networks are called simply Steiner networks.

LEMMA 1.2. Let a Steiner network $N$ has $k$ boundary points. Then $N$ has $(k-2)$ Steıner points and $(2 k-3)$ sides.

Proof. Assume that the network $N$ has $m$ Steiner points and $c$ sides. By calculating we have $k=3 m-2(m-1)$. Hence, we obtain $m=k-2$ and $c=2 k-3$. The lemma is proved.

Definition 1.3. Two boundary points of a Steiner network are called adjacent boundary points if they are ends of two adjacent sides (i.e. sides, which have a common end).

Received July 1, 1993. 
LEMMA 1.4. A Steiner network always has at least two adjacent boundary points.

Proof. Suppose that the given network $N$ has not any two adjacent boundary points. We consider a boundary point $A$. The network $N$ has only one side a with the end $A$. The second end of a is denoted by $A_{1}$. Then $N$ has two sides (different from $a$ ) with the end $A_{1}$. We denote by $B_{1}, B_{2}$ the other ends of the sides $a_{1}, a_{2}$ respectively. Among $B_{1}, B_{2}$ there is at lest one point, for example, $B_{1}$. Further, the $N$ has two sides $b_{1}, b_{2}$ (different from $a_{1}$ ) joining $B_{1}$ with $C_{1}$ and $C_{2}$ respectively (Fig. 1). Go on, because the network $N$ is acyclic we obtain infinite number of Steiner points. That is impossible. The lemma is proved.

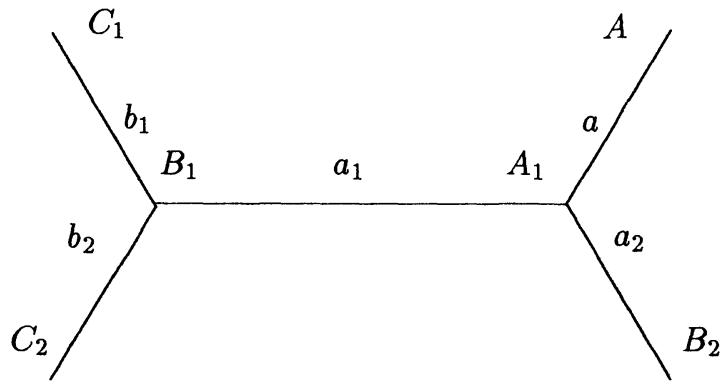

Fig. 1

THEOREM 1.5. Every Steıner network with $k$ boundary points has a finite series of subnetworks $N_{1}, N_{2}, \ldots, N_{k-2}$ such that

$$
N_{1} \subset N_{2} \subset \ldots \subset N_{k-2=N}
$$

Here $N_{1}$ has only one Steiner point, $N_{\imath+1}=N_{\imath} \bigcup\left\{a_{i}, b_{i}\right\} 1 \leq i \leq k-3$ and $a_{i}, b_{i}$ are sides of $N$ that do not belong to $N_{\imath}$.

Proof. Let $a_{k-3}, b_{k-3}$ be sides of $N$ joining two adjacent boundary points. We put $N_{k-3}=N \backslash\left\{a_{k-3}, b_{k-3}\right\}$. Then $N_{k-3}$ is a Steiner network. By Lemma $1.4 N_{k-3}$ has two sides joining adjacent boundary points, for example, $a_{k-4}, b_{k-4}$. We put $N_{k-4}=$ $N_{k-3} \backslash\left\{a_{k-4}, b_{k-4}\right\}$. Go on, after $k-3$ steps we get a series of subnetworks we need.

Definition 1.6. A Steiner network is said to be oriented if its sides can be oriented so that every two adjacent sides are oriented opposite to each other.

\section{Corollary 1.7. A Steıner network has exactly two orientatıons.}

Proof. Suppose that the given network $N$ has $k$ boundary points. By Corollary 1.5 $N$ has a series of subnetworks $N_{1} \subset N_{2} \subset \ldots \subset N_{k}=N$. We consider $N_{1}$. This subnetwork has only one point. Clearly, $N_{1}$ has exactly two following orientations (Fig. 2).

Further, we assume that the subnetwork $N_{b}$ has exactly two orientations and consider the subnetwork $N_{\imath+1}=N_{\imath} \bigcup\left\{a_{i}, b_{i}\right\}$ Suppose that the sides $a_{i}, b_{i}$ meet at $A$ and denote by $c_{i}$ the side in $N_{z}$ with the end $A$. Clearly, each orientation in $c_{i}$, corresponding a certain orientation in $N_{\imath}$, completely defines a orientation on $a_{i}$ and $b_{i}$ (Fig. 3). Thus, 
there are exactly two orientations in $N_{\imath+1}$. Proceeding the process we can prove the corollary.
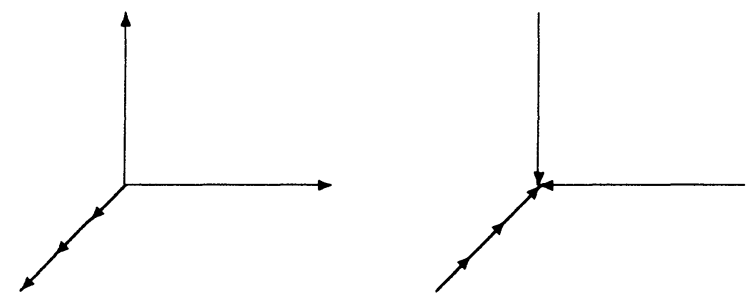

Fig. 2
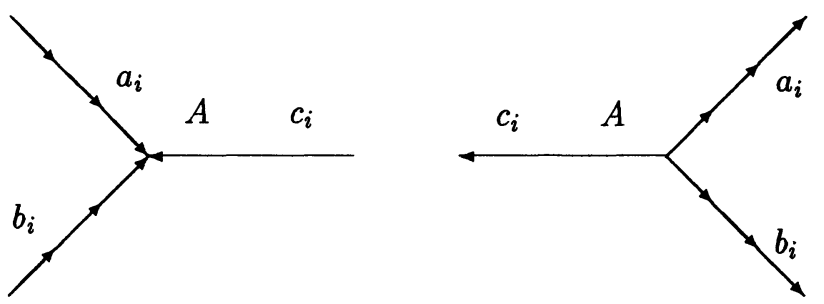

Fig. 3

\section{Globally minimal Steiner networks}

We remember that a path of a network is any continuous series of sides (with orientation) joining two vertices. If these vertices are boundary points then it is called a maximal path. A system of maximal paths is said to be independent if every one of them is not a combination of other paths from the system.

Definition 2.1. A system of mximal paths $\left\{P_{j}\right\}_{j}$ in $N$ is called a basis of maximal paths if it satisfies the following conditions:

(1) The union of all paths from $\left\{P_{\jmath}\right\}_{j}$ overlaps $N$

(2) The system $\left\{P_{j}\right\}_{j}$ is indenpendent

(3) Every maximal path in $N$ is a combination of path from $\left\{P_{\jmath}\right\}_{J}$

THEOREM 2.2. Every oriented Steiner network $N$ with $k$ boundary points has a basis of maximal paths consisting of $(k-1)$ paths.

Proof. We shall prove the theorem by induction for number $k$. At first we consider the case $k=3$, i.e. the given network has three boundary points with three sides $a, b, c$ 
oriented as in Fig. 4.

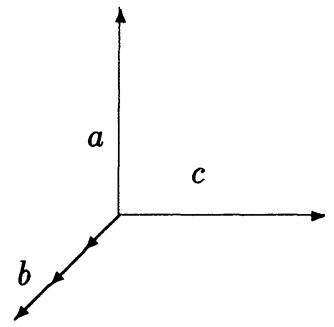

Fig. 4

We put $P_{1}=a-b, P_{2}=c-b$. Clearly, the system $\left\{P_{1}, P_{2}\right\}$ is a basis of maximal paths in $N$. Now, we assume that a Steiner network with i $(i>3)$ boundary points has a basis of maximal paths consisting of $(i-1)$ paths. There is in $N_{2}$ a side $c_{i}$ ended at $A_{2}$. Assume that the side $c_{i}$ belongs to $P_{i_{1}}, \ldots, P_{i_{k}}, 1 \leq i_{1}, \ldots, i_{k} \leq i-1$. We put:

$$
\begin{aligned}
\bar{P}_{\alpha} & =P_{\alpha}, \alpha \neq i_{1}, i_{2}, \ldots, i_{k} \\
\bar{P}_{\imath \jmath} & =P_{i \jmath}-\epsilon_{i j}\left(c_{i}\right) b_{i} \\
\bar{P}_{\imath} & =a_{i}-b_{i}
\end{aligned}
$$

Where $\epsilon_{\imath \jmath}$ is the sign of the side $c_{i}$ in the path $P_{i j}$. To prove that the system $\left\{\bar{P}_{1}, \ldots, \bar{P}_{i}\right\}$ is a basis of maximal paths in $N_{i+1}$ we need only to check the condition (3) in definition 2.1. Let $\bar{P}$ be a path joining two boundary points $A$ and $B$ in $N_{\imath+1}$. The first case: $A, B$ belong to $N_{\imath}$. Suppose that $P$ is a path joining $A, B$ in $N_{\imath}$. Then $P$ is the combination, for example, of $P_{j 1}, \ldots, P_{j m}$ from $\left\{P_{1}, \ldots, P_{i-1}\right\}$.

Hence, $\bar{P}$ is the combination of $\bar{P}_{j 1, \ldots, \bar{P}_{j m}}$ in $N_{i+1}$.

The second case: $A$ belongs to $N_{\imath}, B$ is an end of $a_{i}$. We assume that $\widetilde{P}$ is the path joining $A, A_{\imath}$ in $N_{\imath}$ and $\widetilde{P}$ is the combination of $\bar{P}_{\alpha_{1}}, \ldots, \bar{P}_{\alpha_{n}}$ from $\left\{P_{1}, \ldots, P_{i-1}\right\}$. Then $\bar{P}$ is the combination of $\bar{P}_{\alpha_{1}}, \ldots, \bar{P}_{\alpha_{n}}, \bar{P}_{\imath}$ in $N_{\imath+1}$ The third case: $A$ belongs to $N_{\imath}, B$ is the end of $b_{i}$. We suppose that $P$ is the path joining $A, A_{\imath}$ in $N i$ and $P$ is the combination of $P_{\beta_{1}}, \ldots, P_{\beta_{t}}$ from $\left(P_{1}, \ldots, P_{i-1}\right)$. Then $\bar{P}$ is the combination of $\bar{P}_{\beta_{1}}, \ldots, \bar{P}_{\beta_{t}}$ in $N_{i+1}$. The fourth case: $A$ is the end of $a_{i}$ and $B$ is the end of $b_{i}$. Then $\bar{P}=\bar{P}_{\imath}$. The theorem is proved.

Definition 2.3. Let $N, N^{\prime}$ be Steiner networks in $R^{n}$ with the same boundary points $A_{1}, \ldots, A_{k}$. We say that $N$ and $N^{\prime}$ are of the same topological type if there is a homeomophism $f: R^{n} \longrightarrow R^{n}$ such that $f\left(A_{i}\right)=A_{i}, i=1, \ldots, k$ and $f(N)=N^{\prime}$.

THEOREM 2.4. Let $N$ be an orıented Steiner network with $k$ boundary points in $R^{n}$ and with a basis of maximal paths $\left\{P_{1}, \ldots, P_{k}\right\}$. Suppose that there is a system of closed differential 1-forms $\omega_{1}, \ldots, \omega_{k}$ on $R^{n}$ such that

$$
\left.\sum_{J \in J_{a}} \omega_{j}\left(\epsilon_{j}(a) \vec{N}_{x}\right)=1\right]
$$




$$
\left\|\sum_{J \in J_{a}} \epsilon_{j}(a) \omega_{j}\right\| \leq 1
$$

Where $J_{a}=\left\{j: a \in P_{j}\right\}$ for any side $a \in N$ and $\vec{N}_{x}$ is the unit tangent vector to $N$ at $x \in a$ with the same orientations $a$. Then $N$ is a length-minımizing network in the class of networks with fixed topological type.

Proof. Let $N^{\prime}$ be any Steiner network belonging to the topological type of $N$. Assume that $f: R^{n} \longrightarrow R^{n}$ is homeomophism such that $f(N)=N^{\prime}$ and $f\left(A_{\imath}\right)=A_{\imath}$ for any $i$, where $A_{\imath}$ are the vertices of $N$. The orientation on $N^{\prime}$ is induced by the orientation on $N$ under $f$. We have $\left\{f\left(P_{1}\right), \ldots, f\left(P_{k}\right)\right\}$ is a basis of the maximal paths in $N^{\prime}$. Denote the length of $N$ and $N^{\prime}$ by $|N|,\left|N^{\prime}\right|$ respectively, and put $a^{\prime}=f(a), x^{\prime}=f(x)$. We have

$$
\begin{aligned}
|N| & =\sum_{a \in N}|a|=\sum_{a} \int_{a} \sum_{j \in J_{a}} \omega_{j}\left(\epsilon_{j}(a) \vec{N}_{x}\right) \\
& =\sum_{\jmath} \int_{P_{3}} \omega_{j}=\sum_{\jmath} \int_{P_{j^{\prime}}} \omega_{j} \\
& =\sum_{a^{\prime}} \int_{a^{\prime}} \sum_{j \in J_{a^{\prime}}} \omega_{j}\left(\epsilon_{j}\left(a^{\prime}\right) \vec{N}_{x^{\prime}}^{\prime}\right) \\
& =\sum_{a} \int_{a^{\prime}} \sum_{j \in J_{a}} \omega_{j}\left(\epsilon_{j}(a) \vec{N}_{x^{\prime}}^{\prime}\right) \\
& \leq \sum_{a^{\prime}}\left|\int_{a^{\prime}} 1\right| \\
& =\sum_{a^{\prime}}\left|a^{\prime}\right| \\
& =\left|N^{\prime}\right|
\end{aligned}
$$

Where $P_{\jmath}^{\prime}=f\left(P_{j}\right)$ and $|a|,\left|a^{\prime}\right|$ are the length of $a$ and $a^{\prime}$, respectvely. The theorem is proved.

Such a system $\left\{w_{j}\right\}$ is called a calibration system on $N$.

Now we remember that locally minimal Steiner networks have following properties:

(1) The network consists of strightline segments

(2) At every vertice the segments meet at angles of $120^{\circ}$.

By using theorem 2.4 we obtain the following result.

THEOREM 2.5. Every locally minımal oriented Steiner network is also length-minimizing in the class of the Steiner networks with the same topological type.

Proof. We shall prove this theorem by induction for the number of boundary points of $N$. At first we consider the case when $N$ has only three boundary points $A_{1}, A_{2}, A_{3}$. 
The network is oriented as in Fig. 5.

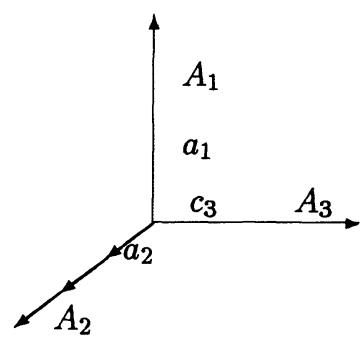

Fig. 5

Denote sides of $N$ by $a_{1}, a_{2}, a_{3}$. We put

$P_{1}=a_{1}-a_{3}$

$P_{2}=a_{2}-a_{3}$

Clearly, the system $\left\{P_{1}, P_{2}\right\}$ is a basis of maximal paths of $N$.

Let $\vec{a}_{1}, \vec{a}_{2}, \vec{a}_{3}$ be the unit tangent vectors to $N$ on $a_{1}, a_{2}, a_{3}$, respectively and $a_{1}^{*}, a_{2}^{*}, a_{3}^{*}$ are the unit co-vectors dual to them. Let $\omega_{1}, \omega_{2}$ be constant differential 1-forms induced by $a_{1}^{*}, a_{2}^{*}$ respectively. Then $\left\{\omega_{1}, \omega_{2}\right\}$ is a calibration system of $N$. Indeed, for any $x \in N$ we have:

If $x \in a_{1}$ then $\omega_{1}\left(\vec{N}_{x}\right)=a_{1}^{*}\left(\vec{a}_{1}\right)=1$

If $x \in a_{2}$ then $\omega_{2}\left(\vec{N}_{x}\right)=a_{2}^{*}\left(\vec{a}_{2}\right)=1$

If $x \in a_{3}$ then $\left(\omega_{1} \omega_{2}\right)\left(\vec{N}_{x}\right)=a_{3}^{*}\left(\vec{a}_{3}\right)=1$

By theorem 2.4 $\mathrm{N}$ is a length-minimizing network.

Further, assume that the statement of the theorem is true for any network with $k$ boundary points and consider an arbitrary locally minimal oriented network $N$ with $k+1$ boundary points.

By Corollary 1.5 we have $N=N_{k} \bigcup\left\{a_{k}, b_{k}\right\}$. Since $N_{k}$ is a locally minimal oriented network with $k$ boundary points there are a basis of maximal paths $\left\{P_{1}, \ldots, P_{k-1}\right\}$ and a calibration system $\left\{\omega_{1}, \ldots, \omega_{k-1}\right\}$ of $N_{k}$ such that

$$
\begin{aligned}
\left\|\sum_{j \in J_{a}} \epsilon_{j}(a) \omega_{j}\right\| & \leq 1 \\
\sum_{j \in J_{a}} \omega_{j}\left(\epsilon_{j}(a) \vec{N}_{x}\right) & =1
\end{aligned}
$$

for any $x \in a \in N$, where $\vec{a}$ denotes the unit tangent vector to a and $a^{*}$ denotes the unit co-vector dual to $\vec{a}$. This means that $\sum_{j} \in J_{a} \epsilon_{j}(a) \omega_{j}=a^{*}$.

Now we choose the basis of maximal paths $\left\{\bar{P}_{1}, \ldots, \bar{P}_{k}\right\}$ of $N$ as in the proof of Theorem 2.2 and let $\omega_{k}$ be the unit constant differential 1-form, dual to $a_{k}$. We shall prove that $\left\{\omega_{1}, \ldots, \omega_{k-1}, \omega_{k}\right\}$ is a calibration system of $N$. Really, denoting by $\bar{\epsilon}_{j}(a)$ the sign of the side a in the path $\bar{P}_{\jmath}$ and putting $\bar{J}_{a}=\left\{j: a \in \bar{P}_{\jmath}\right\}$ we have : 
a. If $x \in a \in N_{k}$ then

$$
\begin{aligned}
\left\|\sum_{j \in \bar{J}_{a}} \bar{\epsilon}_{j}(a) \omega_{j}\right\| & =\left\|\sum_{j \in J_{a}} \epsilon_{j}(a) \omega_{j}\right\|=\left\|a^{*}\right\|=1 \\
\sum_{\jmath \in \bar{J}_{a}} \omega_{j}\left(\bar{\epsilon}_{j}(a) \vec{N}_{x}\right) & =\sum_{j \in J_{a}} \omega_{j}\left(\epsilon_{j}(a) \vec{N}_{x}\right)=1
\end{aligned}
$$

b. If $x \in b_{k}$ then

$$
\begin{aligned}
& \left\|\sum_{\jmath \in \bar{J}_{b_{k}}} \epsilon_{j}\left(b_{k}\right) \omega_{j}\right\|=\left\|-\sum_{\jmath \in J_{c_{k}}} \epsilon_{j}\left(c_{k}\right) \omega_{\jmath}-\omega_{k}\right\|=\left\|-c_{k}^{*}-a_{k}^{*}\right\|=\left\|b_{k}^{*}\right\|=1 \\
& {\left[\sum_{\jmath \in \bar{J}_{b_{k}}} \bar{\epsilon}_{j}\left(b_{k}\right) \omega_{\jmath}\right]\left(\vec{N}_{x}\right)=\left[-\sum_{\jmath \in J_{c_{k}}} \epsilon_{j}\left(c_{k}\right) \omega_{j}-\omega_{k}\right]\left(\vec{N}_{x}\right)} \\
& =\left[-c_{k}^{*}-a_{k}^{*}\right]\left(\vec{b}_{k}\right) \\
& =b_{k}^{*}\left(\vec{b}_{k}\right) \\
& =1
\end{aligned}
$$

c. If $x \in a_{k}$ then

$$
\begin{array}{r}
\left\|\sum_{\jmath \in \bar{J}_{a_{k}}} \bar{\epsilon}_{j}\left(a_{k}\right) \omega_{j}\right\|=\left\|\omega_{k}\right\|=1 \\
\sum_{\jmath \in \bar{J}_{a_{k}}} \bar{\epsilon}_{j}\left(a_{k}\right) \omega_{j}\left(\vec{N}_{x}\right)=\omega_{k}\left(\vec{a}_{k}\right)=1
\end{array}
$$

Thus, we have constructed a calibration system $\left\{\omega_{1}, \ldots, \omega_{k-1}, \omega_{k}\right\}$ of $N$. The theorem is proved.

\section{REFERENCE}

[1] Dao Trong Thi, Minimal real currents on compact Riemannian maanifold, Izv. Akad. Nauk. Ser. Math. 41 (1977), 853-867; English transl., in Math. USSR-Izv. 11 (1977).

[2] Dao Trong Thi, Globally minimal currents and surfaces in Riemannian manifolds, Acta mathematica. No. 2. Vol. 10, 1985.

[3] R. Harvey, H.B. Lawson, Calibrated geometries, Acta Math. 148 (1982), 47-157.

[4] Gary Lawlor, Frank Morgan, Minimizing cones and networks: immiscible fluids, norms and calibrations (Preprint 2/1/1991).

[5] Dao Trong Thi, A. T. Fomenko, Minimal surfaces, stratified multivarifolds and the Plateau problem AMS-Providence-Rhode, Island, 1991.

[6] Ivanov-Tuzhilin, Solution to the Steiner problem for convex boundaries, Vestnik Moskov. Univer. Ser. Math. Mech. No. 2, 1989. 
DaO Trong ThI

HANOI UNIVERSITY

90 - Nguyen Trai Street

Dong Da, HaNoi, VietNam

NGUYen HuU Quang

Department of Mathematics

Pedagogical Institute of Vinh

Vinh CITy, NGhe AN, Vietnam 